American J. of Engineering and Applied Sciences 4 (1): 116-123, 2011

ISSN 1941-7020

(C) 2010 Science Publications

\title{
Simultaneous Thermodynamic and Kinetic Parameters Determination Using Differential Scanning Calorimetry
}

\author{
${ }^{1}$ Nader Frikha, ${ }^{2}$ Mohamed Bouroukba, ${ }^{2}$ Eric Schaer and ${ }^{2}$ Jean-Leon Houzelot \\ ${ }^{1}$ Unit of research: Environment, Catalysis and Processes Analysis, \\ National Engineering School of Gabes, Gabes University, \\ Street Omar Ibn ElKhattab - 6029 Gabes, Tunisia \\ ${ }^{2}$ Laboratory of Reactions and Process Engineering, \\ National School of Chemical Industries, Street Grandville, \\ BP 20451, 54001 Nancy, France
}

\begin{abstract}
Problem statement: The determination of reaction kinetics is of major importance, as for industrial reactors optimization as for environmental reasons or energy limitations. Although calorimetry is often used for the determination of thermodynamic parameters alone, the question that arises is: how can we apply the Differential Scanning Calorimetry for the determination of kinetic parameters. The objective of this study consists to proposing an original methodology for the simultaneous determination of thermodynamic and kinetic parameters, using a laboratory scale Differential Scanning Calorimeter (DSC). The method is applied to the dichromate-catalysed hydrogen peroxide decomposition. Approach: The methodology is based on operating of experiments carried out with a Differential Scanning Calorimeter. The interest of this approach proposed is that it requires very small quantities of reactants (about a few grams) to be implemented. The difficulty lies in the fact that, using such microcalorimeters, the reactants temperature cannot directly be measured and a particular calibration procedure has thus to be developed, to determine the media temperature in an indirect way. The proposed methodology for determination of kinetics parameters is based on resolution of the coupled heat and mass balances. Results: A complete kinetic law is proposed. The Arrhenius parameters are determined as frequency factor $\mathrm{k}_{0}=1.39 \times 109 \mathrm{~s}^{-1}$ and activation energy $\mathrm{E}=$ $54.9 \mathrm{~kJ} \mathrm{~mol}^{-1}$. The measured enthalpy of reaction is $\Delta \mathrm{rH}=-94 \mathrm{~kJ}$ mol-1. Conclusion: The comparison of the results obtained by such an original methodology with those obtained using a conventional laboratory scale reactor calorimetry, for the kinetics determination of, shows that this new approach is very relevant.
\end{abstract}

Key words: Kinetics of reaction, enthalpy of reaction, hydrogen peroxide, Differential Scanning Calorimeter (DSC), laboratory scale

\section{INTRODUCTION}

The determination of process optimal conditions is most important to ensure a process efficient, economical and safe. It can be accomplished by several approaches, among which is the experimental approach. This approach is to study the influence of each process parameter (Cansee et al., 2008). This study may be followed by the development of a software tool, using a control program, to optimize operating conditions in real time (Javanmard et al., 2010). Another approach is to determine the kinetic law and to model the reaction, then easily determine the optimum reaction conditions
(Frikha et al., 2006). On the other hand, the design of a reactor at the industrial scale, for the implementation of an exothermic reaction in batch or continuous mode, requires the precise knowledge of both thermodynamic and kinetic parameters of the chemical reaction. Calorimetry is perhaps one of the oldest scientific known technologies, with published examples dating back to the 18th century (Ubrich et al., 2001; Zogg et al., 2004). If the thermodynamic parameters are generally determined by calorimetry, the much more delicate determination of the reaction's kinetic parameters is frequently omitted. The reasons of this practice are essentially due to economic factors. Indeed,

Corresponding Author: Nader Frikha, Unit of research: Environment, Catalysis and Processes Analysis, ENIG, Street Omar Ibn ElKhattab - 6029 Gabes, Tunisia, Tel: + 21675392100 
a traditional kinetic study is time and labor consuming, on one hand by the development of a reliable analytical protocol and on the other hand by the fact that the implemented reactants are sometimes very expensive. In order to solve these problems, two types of kinetics determination methodologies have been developed in the last decade: one by using calorimetry experiments to fit global kinetics models and the other by carrying out kinetics experiments on very small quantities of reactants.

If the Differential Scanning Calorimeters are well developed for the measurement of heat capacities or heats of reaction for very small quantities of reactants, their major disadvantage is that the temperature inside the measurement vessels cannot be directly measured, what prevents a priori any kinetic measurement. The aim of the present study is to show that a precise kinetic model can however be determined by experiments carried out with a Differential Scanning Calorimeter. This apparatus allows indeed measurements on very small quantities of reactants, what makes it possible to answer the limitation related to the cost of the products. Moreover, it will be shown that the temperature within the measuring vessels, as well as the transfer and capacity parameters of each vessel, can be deduced of the measured signal through a series of calibration procedure, which finally allows determining precisely the kinetic parameters.

This study is applied to the kinetics determination of the dichromate catalyzed hydrogen peroxide decomposition. The comparison between the measurements carried out using the DSC and those carried out on a small laboratory scale reactor testify for the accuracy of the methodology, after a scaling down factor of more than 100 .

\section{Calorimeter calibration:}

Calorimeter description: The Differential Thermal Analyses (DTA) was performed with a SETARAM C80 Differential Scanning Calorimeter (microDSC III) of the Calvet type. Calvet calorimeters are differential devices that use high performance thermopiles that completely surround the area where the thermal phenomenon occurs. Two thermopiles respectively enclose a measurement vessel (useful volume of 12 $\mathrm{cm}^{3}$ ), in which the sample to be studied is placed and a reference vessel containing a thermally inert product. The two piles are assembled in opposition to form a fluxmeter which indicates the difference of the heat flow exchanged by each vessel and their surroundings.

This apparatus makes it possible to follow chemical reactions by putting into contact two reactants, initially contained in two compartments separated by a membrane. The reaction is initiated, once thermal equilibrium is reached, by performing the membrane using an agitator as can be seen in Fig. 1.

The traditional use of DSC is for the measurement of heat-storage capacities and or enthalies of reactions. These quantities are determined by total heat balances between two defined (initial and final) thermal equilibria. On the other hand, DSCs are practically never used for the measurement of reaction kinetics because the direct measurement of the temperature of the reactional medium is impossible. The originality of the work presented in this article thus consists in describing a nonconventional method of calibration which makes it possible to estimate the temperature inside the measurement vessel using an indirect way. It depends in particular on the modeling of the dynamic response of the calorimeter when subjected to a thermal disturbance.

Relation between heat flow and measured signal: The heat flow exchanged between each vessel and their surrounding is related to the electric potential difference E delivered by the corresponding thermopile. Thus, affecting respectively indices meas and ref to the measuring and reference vessels, the heat fluxes exchanged by each cell can be expressed as:

$$
\begin{aligned}
& \varphi_{\text {meas }}=U_{\text {meas }} A_{\text {meas }}\left(T_{e}-T_{\text {meas }}\right)=\sigma_{\text {meas }} E_{\text {meas }} \\
& \varphi_{\text {ref }}=U_{\text {ref }} A_{\text {ref }}\left(T_{e}-T_{\text {ref }}\right)=\sigma_{\text {ref }} E_{\text {ref }}
\end{aligned}
$$

Where:

$\mathrm{T}_{\mathrm{e}} \quad=$ The surrounding temperature (which can be monitored and regulated)

$\mathrm{T}_{\text {meas }}$ and $\mathrm{T}_{\mathrm{ves}}=$ The temperatures (that are not measured) inside each vessel

The calorimeter is designed in such a way that the two vessels behave in a symmetrical way so that $\sigma_{\text {ref }}$ and $\sigma_{\text {meas }}$ are identical. Thus, the electric signal delivered by the calorimeter is a tension proportional to the difference in the heat flows between the two vessels:

$\Delta \varphi=\varphi_{\text {meas }}-\varphi_{\text {ref }}=\sigma\left(\mathrm{E}_{\text {meas }}-\mathrm{E}_{\text {ref }}\right)=\sigma \Delta \mathrm{E}$

The value of the coefficient $\sigma$ is determined using a thermal source (an electric resistance) of known power. For the performed experiments, a value of $\sigma=3.33 .10^{-2}$ $\mathrm{mW} . \mu \mathrm{V}^{-1}$ for a temperature of $25^{\circ} \mathrm{C}$ has been determined.

The calorimeter calibration is then carried out in several steps 


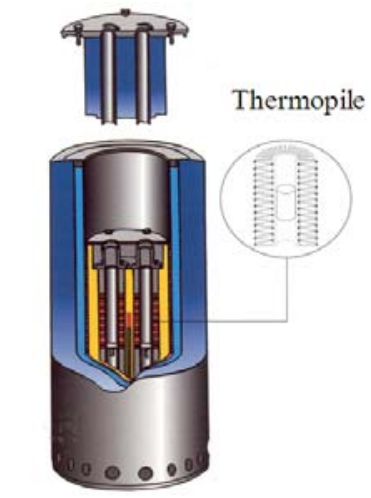

Setaram C 80 DSC

Fig. 1: Experimental device

\section{MATEIALS AND METHODS}

Vessel heat capacity: Initially, only one empty vessel is placed in the calorimeter and a linear surrounding temperature variation between two initial and final temperatures $T_{e}$,init and $T_{e}$,fin is applied. These first experiments make it possible to measure the heatstorage capacity of the vessel and to check for the symmetry of the calorimeter. The input (surrounding temperature $T_{e}$ Vs time) and output (measured heat flow $\Delta \phi$ Vs time) signals for such an experiment are presented in Fig. 2. The excellent symmetry of the device can be Remarque.

These results show that both vessels behave like a first order system. Indeed, the theoretical output signal of a first order system submitted to a linear variation of the input signal is, after a limited period, a linear variation of the same slope than the input signal. During this last step, the difference in temperature between the vessels and their surrounding remains constant, what leads to a constant measured signal on the DSC, which can easily be observed in Fig. $2 b$.

A heat balance on each empty vessel leads to:

$\mathrm{UA}\left(\mathrm{T}_{\mathrm{e}}-\mathrm{T}_{\mathrm{ves}}\right)=\mathrm{mC}_{\mathrm{pves}} \frac{\mathrm{dT}_{\mathrm{ves}}}{\mathrm{dt}}=\Delta \varphi$

where, $T_{\text {ves }}$ is the vessel temperature. After integration, relation 3 gives:

$$
\mathrm{mC}_{\mathrm{pves}} \int_{\mathrm{T}_{\text {init }}}^{\mathrm{T}_{\text {fin }}} \mathrm{dT}_{\mathrm{ves}}=\int_{0}^{\infty} \Delta \varphi=\mathrm{mC}_{\mathrm{pves}}\left(\mathrm{T}_{\mathrm{e}, \text { fin }}-\mathrm{T}_{\mathrm{e}, \text { init }}\right)
$$

The numerical integration of the output signal $\Delta \phi$ leads to two values of $\mathrm{mC}_{\mathrm{p}}$ for both vessels, respectively equal to 36.9 and 37.1 J.K ${ }^{-1}$.

Heat transfer coefficient: The second calibration step consists in placing the two vessels in their respective

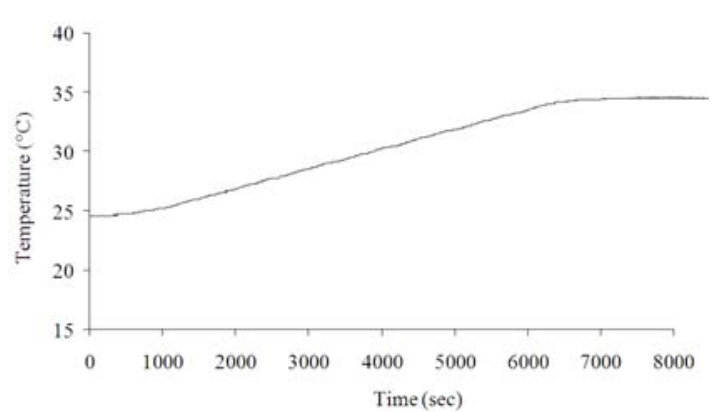

(a)

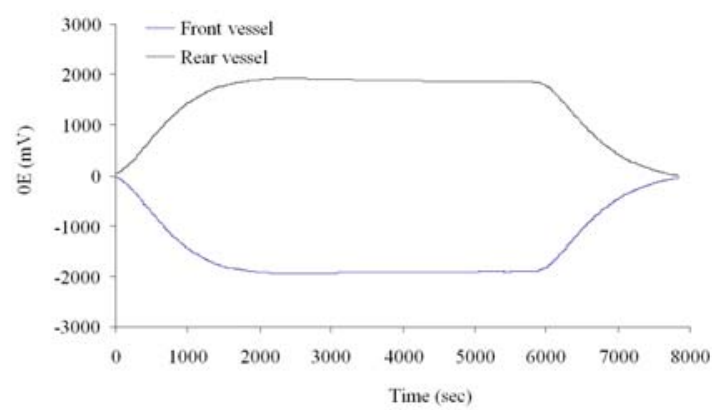

(b)

Fig. 2: Heat capacity measurement. Input (top) and output (bottom) signals

location in the DSC. One is empty and the other contains the hydrogen peroxide solution without catalyst so that the reaction does not happen. Affecting reac and ves indices to reacting (or measuring) and empty vessels respectively and assuming that the thermal transfer resistance is localized between the vessel and the thermopile, meaning identical temperatures for the empty reference and measuring vessels, a heat balance on each vessel subjected to a linear temperature variation leads to:

$$
\begin{aligned}
\varphi_{\text {ves }} & =\mathrm{U}_{\text {ves }} \mathrm{A}_{\text {ves }}\left(\mathrm{T}_{\mathrm{e}}-\mathrm{T}_{\mathrm{ves}}\right) \\
& =\mathrm{mC}_{\mathrm{pves}} \frac{\mathrm{dT} \mathrm{T}_{\mathrm{ves}}}{\mathrm{dt}}=\sigma_{\text {ves }} \mathrm{E}_{\mathrm{ves}} \\
\varphi_{\text {reac }} & =\mathrm{U}_{\text {reac }} \mathrm{A}_{\text {reac }}\left(\mathrm{T}_{\mathrm{e}}-\mathrm{T}_{\text {reac }}\right) \\
& =\left(\mathrm{mC}_{\mathrm{p} \text { ves }}+\mathrm{mC}_{\text {preac }}\right) \frac{\mathrm{dT}_{\text {reac }}}{\mathrm{dt}}=\sigma_{\text {reac }} \mathrm{E}_{\text {reac }}
\end{aligned}
$$

As it has been verified that both vessels behave in a symmetrical way, we make the assumption that the heat transfer coefficient $U$, the exchange area $A$ and the proportionality coefficient $\sigma$ are identical for the two vessels. It can then be deduced:

$$
\begin{gathered}
\Delta \varphi=\sigma \Delta \mathrm{E}= \\
\mathrm{UA}\left(\mathrm{T}_{\text {ves }}-\mathrm{T}_{\text {reac }}\right)=\left(\mathrm{mC}_{\mathrm{pves}}+\mathrm{mC}_{\text {preac }}\right) \\
\frac{\mathrm{dT}_{\text {reac }}}{\mathrm{dt}}-\mathrm{mC}_{\text {pves }} \frac{\mathrm{dT}_{\text {ves }}}{\mathrm{dt}}
\end{gathered}
$$


If the surrounding (or thermopile) temperature follows a linear variation, according to:

$$
\mathrm{T}_{\mathrm{e}}=\mathrm{T}_{\mathrm{e}, \text { init }}+\mathrm{St}
$$

The integration of the heat balances (relations 5) leads to:

$$
\begin{aligned}
& \mathrm{T}_{\text {ves }}=\mathrm{T}_{\text {init }}+\mathrm{St}-\mathrm{c}(\phi-1) \tau\left[1-\exp \left(-\frac{\mathrm{t}}{(\phi-1) \tau}\right)\right] \\
& \mathrm{T}_{\text {reac }}=\mathrm{T}_{\text {init }}+\mathrm{St}-\mathrm{c} \phi \tau\left[1-\exp \left(-\frac{\mathrm{t}}{\phi \tau}\right)\right]
\end{aligned}
$$

with, as usage in calorimetry:

$$
\phi=\frac{\mathrm{mC}_{\mathrm{pves}}+\mathrm{mC}_{\mathrm{preac}}}{\mathrm{mC}_{\text {preac }}} \text { and } \tau=\frac{\mathrm{mC}_{\mathrm{preac}}}{\mathrm{UA}}
$$

The thermal inertia and thermal time constant respectively.

The difference between both expressions of relations 8 gives:

$$
\begin{aligned}
\Delta \varphi & =\sigma \Delta \mathrm{E}=\mathrm{UA}\left(\mathrm{T}_{\text {ves }}-\mathrm{T}_{\text {reac }}\right) \\
& =\mathrm{UAS} \tau\left[\begin{array}{l}
1+(\phi-1) \exp \left(-\frac{\mathrm{t}}{(\phi-1) \tau}\right) \\
-\phi \exp \left(-\frac{\mathrm{t}}{\phi \tau}\right)
\end{array}\right]
\end{aligned}
$$

Once the temperature variation begins, for observation times much higher than the characteristic time $\tau$ (at least 5 times larger than $\tau$ ) it can be remarqued that the exponential term becomes negligible in front of 1 . Thus the temperatures of the two vessels increase linearly against time and consequently their difference leads to:

$$
\mathrm{T}_{\mathrm{ves}}-\mathrm{T}_{\text {reac }}=\frac{\Delta \phi}{\mathrm{UA}}=\mathrm{S} \tau
$$

which finally gives:

$$
\Delta \phi=\mathrm{S}_{\mathrm{m}} \mathrm{m}_{\text {preac }}
$$

When the value of $\Delta \phi$ becomes stationary, the knowledge of the value of the slope $\mathrm{S}$ makes it possible to evaluate the product $\mathrm{mC}_{\text {preac }}$.

Regarding the transitory part of the output signal, the comparison of the measured signal with the mathematical expression (Eq. 10) allows, by nonlinear regression, to identify the value of $\tau$. Finally, knowing $\mathrm{mC}_{\text {preac }}$ and $\tau$, the value of UA can easily be estimated and consequently the temperature difference between the two vessels can be evaluated.

\section{RESULTS AND DISCUSSION}

To carry out the calibration procedure, 4.6 grams of solution were loaded in the measuring vessel. Assuming identical physical properties as that of water, this quantity of solution may have a $\mathrm{mC}_{\text {preac }}$ of 19.25 J.K ${ }^{-1}$. When a linear temperature variation of $0.1 \mathrm{~K}$ $\mathrm{min}^{-1}$ is then imposed, the variations of the temperature and of the measured signal $\Delta \phi$ against time are presented in Fig. 3.

The constant heat flow difference value during most of the temperature rise (between 2500 to more than $6000 \mathrm{sec}$ ) can be remarqued in Fig. 3b. Knowing this value $\Delta \phi=32.1 \mathrm{~mW}$, it can easily be deduced:

$\mathrm{mC}_{\text {preac }}=\frac{\Delta \varphi}{\mathrm{S}}=\frac{0.0321}{0.1 / 60}=19.28 \mathrm{~J} . \mathrm{K}^{-1}$

This last value is very close to that calculated the properties of water. This measurement leads to a value of the thermal inertia $\varphi$ equal to 2.92 .

When, using nonlinear regression, the transitory parts of the measured signal (Fig. 3b) is fitted by relation (10), a value of the thermal time constant $\tau$ equal to $219 \mathrm{~s}$ can be estimated. The comparison of the measured and calculated signal is presented in Fig. 4, which testifies for the accuracy of the calculation.

Thus, the product UA is $8.810^{-2} \mathrm{~W} . \mathrm{K}^{-1}$, which finally makes it possible to estimate the temperature difference between the two vessels by the relation:

$\Delta \mathrm{T}=\mathrm{T}_{\text {ves }}-\mathrm{T}_{\text {reac }}=\frac{\Delta \varphi}{\mathrm{UA}}=\frac{\Delta \varphi}{0.088}$

where, $\Delta \phi$ is expressed in $\mathrm{W}$.

Application to the measurement of reaction kinetics: This methodology is applied to the measurement of kinetics of the dichromate catalyzed hydrogen peroxide decomposition. This reaction was the subject of some previous studies (Korneeva et al., 1972; Kobozev et al., 1972; Brungs et al., 1988) and involves the following stoechiometric mechanism:

$$
\begin{aligned}
2 \mathrm{H}_{2} \mathrm{O}_{2}+\mathrm{Cr}_{2} \mathrm{O}_{7}^{2-} & \leftrightarrow \mathrm{Cr}_{2} \mathrm{O}_{9}^{2-}+2 \mathrm{H}_{2} \mathrm{O} \\
\mathrm{Cr}_{2} \mathrm{O}_{9}^{2-} & \rightarrow \mathrm{Cr} 2 \mathrm{O}_{7}^{2-}+\mathrm{O}_{2}
\end{aligned}
$$




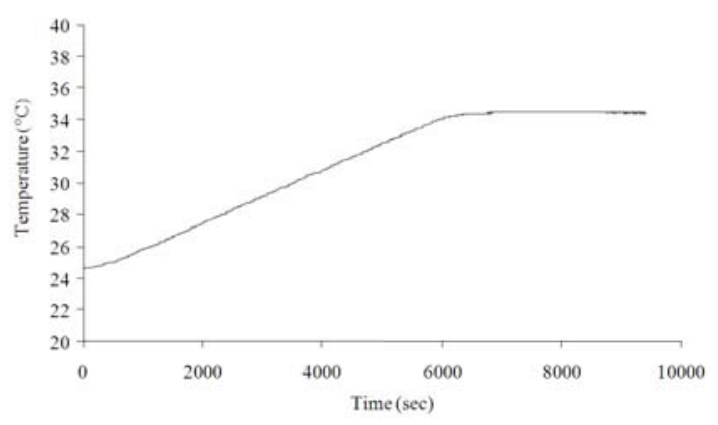

(a)

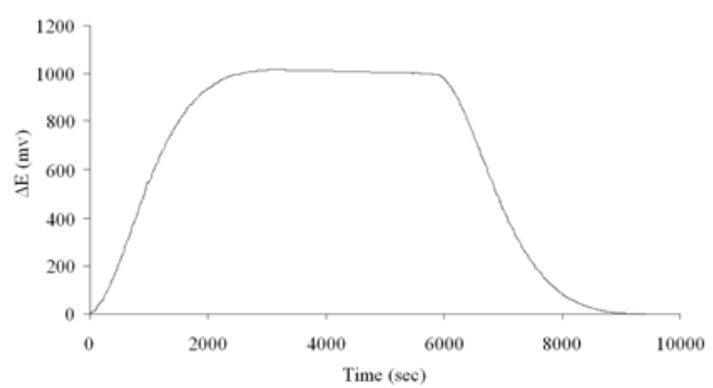

(b)

Fig. 3: Measuring vessel calibration. Temperature variation (top) and measured heat flow (bottom) against time

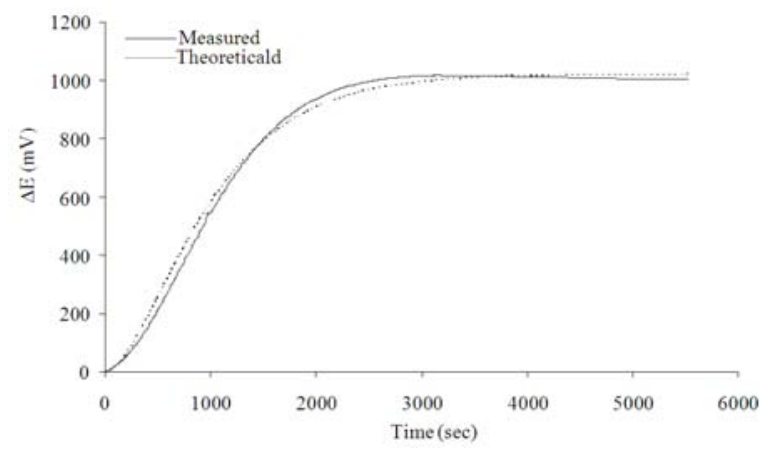

Fig. 4:Comparison between experimental (continuous line) and optimized (dashed line) signals

It is supposed that the first reactional stage is fast and balanced and the second slower one constitutes the determining kinetic stage (Frugoni et al., 1986).

Equilibrium constant $\mathrm{K}$ and reaction rate $\mathrm{r}_{\mathrm{cr}}$ can be expressed as follows:

$$
\begin{aligned}
& \mathrm{K}=\frac{\left[\mathrm{Cr}_{2} \mathrm{O}_{9}^{2-}\right]\left[\mathrm{H}_{2} \mathrm{O}\right]^{2}}{\left[\mathrm{Cr}_{2} \mathrm{O}_{7}^{2-}\right]\left[\mathrm{H}_{2} \mathrm{O}_{2}\right]^{2}} \\
& \mathrm{r}_{\mathrm{Cr}}=\mathrm{k}^{\prime}\left[\mathrm{Cr}_{2} \mathrm{O}_{9}^{2-}\right]
\end{aligned}
$$

Defining K' as:

$$
\mathrm{K}^{\prime}=\frac{\mathrm{K}}{\left[\mathrm{H}_{2} \mathrm{O}\right]^{2}}
$$

The consumption rate of dichromate, which is half of that of peroxide, can finally be expressed as:

$$
\begin{aligned}
\mathrm{r}_{\mathrm{H}_{2} \mathrm{O}_{2}}=2 \mathrm{r}_{\mathrm{Cr}} & =2 \mathrm{k}^{\prime}\left[\mathrm{Cr}_{2} \mathrm{O}_{9}^{2-}\right]=\mathrm{k}\left[\mathrm{Cr}_{2} \mathrm{O}_{9}^{2-}\right] \\
& =\mathrm{k} \frac{\mathrm{K}^{\prime}\left[\mathrm{H}_{2} \mathrm{O}_{2}\right]^{2}\left[\mathrm{Cr}_{2} \mathrm{O}_{7}^{2-}\right]_{0}}{1+\mathrm{K}^{\prime}\left[\mathrm{H}_{2} \mathrm{O}_{2}\right]^{2}}
\end{aligned}
$$

According to experimental conditions, the apparent order in peroxide presents two limiting cases: at the beginning of the reaction the peroxide concentration is high, the kinetics does not depend on this concentration and presents a zeroth order in peroxide:

$\mathrm{K}^{\prime}\left[\mathrm{H}_{2} \mathrm{O}_{2}\right]^{2}>>1$ then $\mathrm{r}=\mathrm{k}\left[\mathrm{Cr} 2 \mathrm{O}_{7}^{2-}\right]_{0}$

At the end of the reaction, the peroxide concentration become weak and the kinetics presents a second order in peroxide:

$\mathrm{K}^{\prime}\left[\mathrm{H}_{2} \mathrm{O}_{2}\right]^{2} \ll<1$ then $\mathrm{r}=\mathrm{kK}{ }^{\prime}\left[\mathrm{H}_{2} \mathrm{O}_{2}\right]^{2}\left[\mathrm{Cr}^{2} \mathrm{O}_{7}^{2-}\right]_{0}$

A preliminary kinetic study (Frikha et al., 2005) allowed determining the kinetic and thermodynamic parameters of this reaction:

$$
\begin{aligned}
\mathrm{k} & =1.39 .10^{9} \exp \left(-\frac{6600}{\mathrm{~T}}\right) \\
\Delta_{\mathrm{r}} \mathrm{H} & =-94 \mathrm{~kJ} \cdot \mathrm{mol}^{-1} \\
\mathrm{~K}^{\prime} & =\exp \left(\frac{2768}{\mathrm{~T}}-5.16\right) \mathrm{L}^{2} \cdot \mathrm{mol}^{-2}
\end{aligned}
$$

Experimental implementation: In each vessel of the DCS, a predetermined quantity of hydrogen peroxide solution is loaded, while the catalyst is added in only one of the vessel and is, before the reaction begins, separated of the reactional medium by a small membrane. It is important to introduce in each vessel a same quantity of reactional media in order to respect the symmetry of the heat capacities of the measuring devices. Once initial thermal balance is reached, the membrane is broken with a small agitator, which initiates the reaction. The analyses can be performed in two modes: isoperibolic mode, which means that the 
external temperature $T_{e}$ is unchanged during the measurement, or in Appling a linear variation of $\mathrm{T}_{\mathrm{e}}$.

Heat and mass balances: The heat balance for the reference vessel is:

$$
\mathrm{T}_{\mathrm{e}}-\mathrm{T}_{\mathrm{ves}}=\phi \tau \frac{\mathrm{dT}_{\mathrm{ves}}}{\mathrm{dt}}
$$

It has been choosen to define conversion $\mathrm{X}$ according to hydrogen peroxide, since dichromate is regenerated as reaction proceeds, as shown by the reactional mechanism. $\Delta \mathrm{T}_{\mathrm{ad}}$ is the adiabatic temperature rise:

$$
\begin{aligned}
{\left[\mathrm{H}_{2} \mathrm{O}_{2}\right] } & =\left[\mathrm{H}_{2} \mathrm{O}_{2}\right]_{\text {init }}(1-\mathrm{X}) \\
\Delta \mathrm{T}_{\text {ad }} & =-\frac{\left[\mathrm{H}_{2} \mathrm{O}_{2}\right]_{\text {init }}\left(-\Delta_{\mathrm{r}} \mathrm{H}\right)}{\rho_{\text {reac }} \mathrm{C}_{\mathrm{p}_{\text {reac }}}}
\end{aligned}
$$

The mass and heat balance for the reacting, or measuring vessel, here become:

$$
\begin{aligned}
& \mathrm{r}=-\frac{\mathrm{d}\left[\mathrm{H}_{2} \mathrm{O}_{2}\right]}{\mathrm{dt}}=\left[\mathrm{H}_{2} \mathrm{O}_{2}\right]_{\text {init }} \frac{\mathrm{dX}}{\mathrm{dt}} \\
& \mathrm{UA}\left(\mathrm{T}_{\mathrm{e}}-\mathrm{T}_{\text {reac }}\right)= \mathrm{mC}_{\text {preac }} \phi \frac{\mathrm{dT}_{\text {reac }}}{\mathrm{dt}} \\
& \mathrm{rV}\left(-\Delta_{\mathrm{r}} \mathrm{H}\right)
\end{aligned}
$$

Which, after reorganization, gives:

$$
\begin{gathered}
\mathrm{T}_{\mathrm{e}}-\mathrm{T}_{\text {reac }}=\phi \tau \frac{\mathrm{dT}_{\text {reac }}}{\mathrm{dt}}+\frac{\left[\mathrm{H}_{2} \mathrm{O}_{2}\right]_{\text {init }} \mathrm{V} \Delta_{\mathrm{r}} \mathrm{H}}{\mathrm{mC}_{\text {preac }}} \\
\tau \frac{\mathrm{dX}}{\mathrm{dt}}=\phi \tau \frac{\mathrm{dT}_{\text {reac }}}{\mathrm{dt}}+\Delta \mathrm{T}_{\text {adia }} \tau \frac{\mathrm{dX}}{\mathrm{dt}}
\end{gathered}
$$

The difference between relations 24 and 20 leads to:

$$
\Delta \mathrm{T}=\mathrm{T}_{\text {ves }}-\mathrm{T}_{\text {reac }}=-\phi \tau \frac{\mathrm{d} \Delta \mathrm{T}}{\mathrm{dt}}+\Delta \mathrm{T}_{\text {adia }} \tau \frac{\mathrm{dX}}{\mathrm{dt}}
$$

And the mass balance in former relation can be expressed as:

$$
\begin{aligned}
\frac{\mathrm{dX}}{\mathrm{dt}}= & \mathrm{A}_{0} \exp \left(-\frac{\mathrm{E}}{\mathrm{RT}_{\text {reac }}}\right) \\
& \frac{\mathrm{K}^{\prime}\left[\mathrm{H}_{2} \mathrm{O}_{2}\right]_{\text {init }}\left[\mathrm{Cr}_{2} \mathrm{O}_{7}^{2-}\right]_{\text {init }}(1-\mathrm{X})^{2}}{1+\mathrm{K}^{\prime}\left[\mathrm{H}_{2} \mathrm{O}_{2}\right]_{\text {init }}^{2}(1-\mathrm{X})^{2}}
\end{aligned}
$$

The resolution of this last equation requires an expression for the variation of $\mathrm{T}_{\text {reac }}$ againt operating conditions. It is obtained by the expression between $\mathrm{T}_{\text {reac }}, \mathrm{T}_{\mathrm{ves}}$ and $\Delta \phi$ using relation (14), which gives for an isoperibolic mode:

$$
\mathrm{T}_{\text {reac }}=\mathrm{T}_{\mathrm{ves}}-\Delta \mathrm{T}=\mathrm{T}_{\mathrm{e}, \text { init }}-\frac{\Delta \varphi}{0.088}
$$

And for a linear variation of the imposed temperature, after resolution of relation (20):

$$
\begin{gathered}
\mathrm{T}_{\text {reac }}=\mathrm{T}_{\text {ves }}-\Delta \mathrm{T}+=\mathrm{T}_{\mathrm{e}, \text { init }}+\mathrm{St}-\mathrm{S} \phi \tau \\
{\left[1-\exp \left(-\frac{\mathrm{t}}{\phi \tau}\right)\right]-\frac{\Delta \varphi}{0.088}}
\end{gathered}
$$

Experimental results: For the presented example, the experimental conditions are: initial quantity of hydrogen peroxide $\mathrm{n}_{2} \mathrm{O}_{2}{ }^{\text {init }}=2.85 .10^{-3} \mathrm{~mol}$, a temperature variation rate of $0.15 \mathrm{~K} . \mathrm{mn}^{-1}$ and an initial temperature of $20^{\circ} \mathrm{C}$. The measured heat flux difference indicated by the DSC, expressed in $\mathrm{mW}$, is presented in Fig. 5.

First of all, integration of the curve presented in Fig. 5 makes it possible to evaluate the heat quantity produced by the reaction. The numerical value is $263 \mathrm{~J}$ whereas a direct calculation using the quantity of introduced peroxyde and the already known enthalpy of reaction leads to $268 \mathrm{~J}$ The agreement is thus excellent.

The measured heat flux difference allows calculating the temperature variation inside the reactional vessel against time, using relation (25), as well as the temperature difference $\Delta \mathrm{T}$ between the measuring and reference vessel, using relation (14).

In order to check the relevance of this methodology to evaluate reaction kinetics, we did not directly try to identify the parameters of kinetic law using the temperature profiles. Indeed, we have already described in a former paper (Frikha et al., 2005) an installation made up of two laboratory scale reactors (heat jacketed reactors of a few hundred milliliters) which are placed in parallel to operate in a similar way to the vessels of a DSC: One in which the reaction is carried out, the other one being used as reference. The advantage of such a configuration is that the temperature can easily be measured inside and outside (in the jacket) each reactor. When implementing under identical experimental conditions, i.e., with a linear variation of the external temperature, the dichromate catalysed hydrogen peroxide decomposition in such a device, we have been able to recompute, using a differential thermal analysis approach, the kinetics of the reaction with a very good accuracy. 


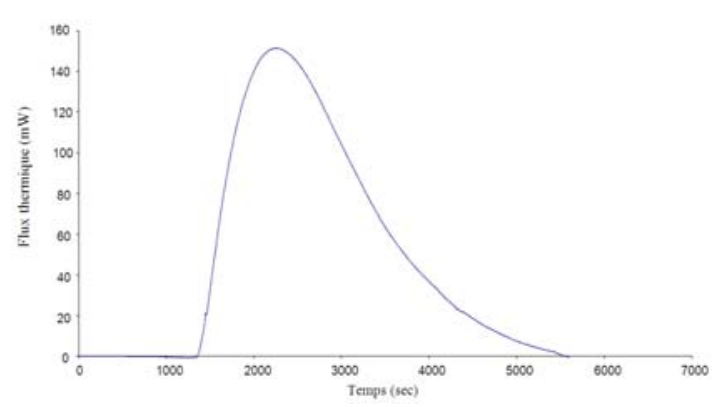

Fig. 5: Heat flow indicated by the DSC for kinetics identification

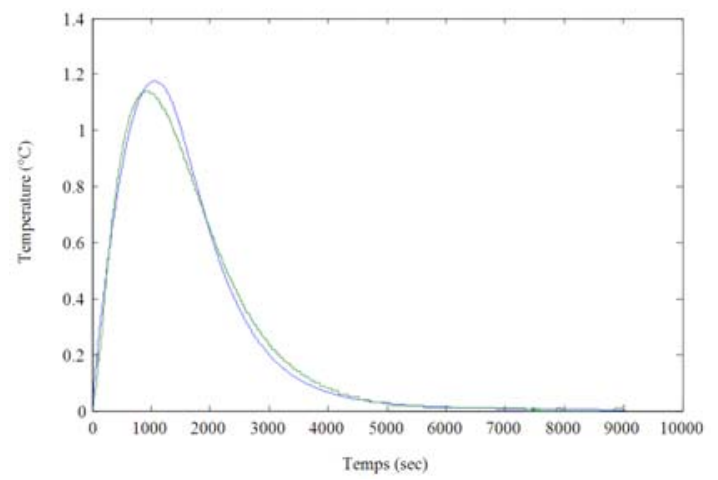

Fig. 6: Comparison between measured and simulated temperature differences

We do not develop here the analysis presented in the preceding article (Frikha et al., 2005). In order to check the relevance of the use of microcalorimetry to quantify kinetic parameters, we use the already determined kinetic law of the reaction to calculate, solving the system of differential equations composed by relations 21 and 23 , the reactional temperature or temperature difference which one should theoretically observe. Figure 6, on which are represented both calculated and measured (using relation 14) temperature difference, shows that the agreement is very satisfactory. We have thus shown that the temperature difference between the measuring and reference vessels of the DSC can easily be deduced of the measured heat flow. It is then possible to compute the temperature evolution in the measuring vessel or to quantify, with a good accuracy, the reactional kinetic parameters. This finally demonstrates that DSC apparatuses are well adapted to the precise measurement of kinetics on very small quantity of reactants.

\section{CONCLUSION}

We have thus demonstrated that the microcalorimetry of laboratory is adapted, since it is proceeded to a meticulousness calibration procedure, for the determination of thermodynamic and kinetics parameters of chemical reactions. The advantage of this methodology is that it requires very small quantity of reactants (about a few grams). Its interest is thus obvious when the reactants are very expensive on one hand and/or when the studied reaction is potentially dangerous on another hand.

\section{REFERENCES}

Brungs, M.P., B.G. Madden and P.L. Seage, 1988, Simulation and experimental studies of metastable states of peroxide reactions in stirred reactors. Chem. Eng. Sci., 43: 2451-2455. DOI: 10.1016/0009-2509(88)85179-0

Cansee, S., J. Uriyapongson, C. Watyotha, T. Thivavarnvongs and J. Varith, 2008, Amphoteric starch in simultaneous process preparation with boxbehnken design for optimal conditions. Am. J. Applied Sci., 5: 1535-1542. DOI: 10.3844/ajassp.2008.1535.1542

Frikha, N., E. Schaer and J.L. Houzelot, 2005. Study of the dichromate-catalysed hydrogen peroxide decomposition: Comparison of different batch mode reactors for the kinetics determination. Thermochimica Acta, 437: 48-58. DOI: 10.1016/j.tca.2005.06.017

Frikha, N., E. Schaer and J.L. Houzelot, 2006. Methodology of multiphase reaction kinetics and hydrodynamics identification: Application to catalyzed nitrobenzene hydrogenation. Chem. Eng. J., 124: 19-28. DOI: 10.1016/j.cej.2006.08.012

Frugoni, J.A.C., M. Zepka, A.R. Figueira and A.S. Campos, 1986. An experiment of homogeneous catalysis. J. Chem. Educ., 63: 549-549. DOI: 10.1021/ed063p549

Javanmard, M., J. Endan, K.A. Abbas and F. Arvin, 2010. Development of a semi-industrial multi fruit dryer system using simultaneous intelligent control. Am. J. Applied Sci., 7: 160-166. DOI: 10.3844/ajassp.2010.160.166

Kobozev, N.I., V.I. Shekhobalova and N.I. Korneeva, 1972. Intermediate products in the homogeneous decomposition of hydrogen peroxide by potassium dichromate. Russian J. Phys. Chem., 46: 840-842.

Korneeva, N.I., V.I. Shekhobalova and N.I. Kobozev, 1972. Kinetics of the decomposition of concentrated hydrogen peroxide solutions by potassium dichromate in a neutral medium. Russian J. Phy. Chem., 46: 210-212. 
Ubrich, O., B. Srinivasan, P. Lerena, D. Bonvin and F. Stoessel, 2001. The use of calorimetry for online optimisation of isothermal semi-batch reactors. Chem. Eng. Sci. 56: 5147-5156. DOI: 10.1016/S0009-2509(01)00183-X
Zogg, A, F. Stoessel, U. Fischer and K. Hungerbuhler, 2004. Isothermal reaction calorimetry as a tool for kinetic analysis. Thermochim. Acta, 419: 1-17. DOI: $10.1016 /$ j.tca.2004.01.015 